\title{
Liver anatomy, intrahepatic vascular and biliary branching system of the mole rat (Spalax leucodon)
}

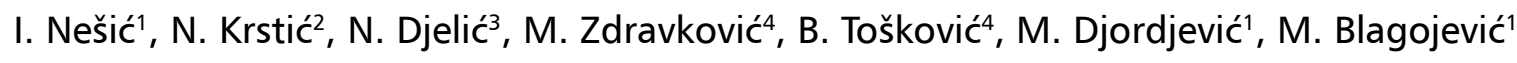 \\ ${ }^{1}$ Department of Anatomy, Faculty of Veterinary Medicine, University of Belgrade, Serbia \\ 2Department of Radiology and Radiation Hygiene, Faculty of Veterinary Medicine, University of Belgrade, Serbia \\ ${ }^{3}$ Department of Biology, Faculty of Veterinary Medicine, University of Belgrade, Serbia \\ ${ }^{4}$ Faculty of Medicine, University of Belgrade, Medical Centre Bežanijska Kosa, Belgrade, Serbia
}

[Received: 24 August 2020; Accepted: 4 October 2020; Early publication date: 27 October 2020]

Background: There are many studies on the morphology of the liver and its blood vessels in experimental animals, but such studies are lacking in the mole rat (Spalax leucodon). The aim of this paper was a detailed basic study on the topography, morphology, vascular and biliary branching systems of the liver in the mole rat.

Materials and methods: Coloured gelatine and mixture of coloured lead oxide and linseed oil were injection contrast masses used to obtain vascular and biliary branching pattern in the liver. It was revealed that the liver of the mole rat had five lobes (left, quadrate, right medial, right lateral and caudate lobes).

Results: The left, undivided lobe was the largest lobe of the liver. The quadrate lobe was divided into two components by a deep notch. The gallbladder, of cylindrical shape, was present and attached to the quadrate lobe. The common bile duct was formed by the union of the left and right hepatic ducts. The pancreatic duct joined the common bile duct before it entered the duodenum. In the present study, only the right medial lobe and quadrate lobe always showed a single lobar artery, portal and hepatic veins. The left lobe showed four lobar arteries, portal and hepatic veins. The caudate lobe with its two processes and the right lateral and medial lobes had different arterial and portal blood supply as well as hepatic and biliary drainage of these lobes. The intrahepatic branches of the proper hepatic artery ran parallel to the branches of the common portal vein in the same lobes of the liver.

Conclusions: The results of this study are significant for comparative studies among different species of rodents and other experimental animals. Morphology, vasculature and biliary tract of the liver in the mole rat were similar to that of other experimental animals and identified differences may be related to the adaptation to the mode of life and diet of this rodent. (Folia Morphol 2021; 80, 4: 888-894)

Key words: liver, a. hepatica propria, v. portae, vv. hepaticae, bile ducts 


\section{INTRODUCTION}

The mole rat (Spalax leucodon) is a rodent belonging to the Muroidea superfamily and the Spalacidae family. Spalax leucodon is the only species of mole rats found in Serbia in the area of Vojvodina province [6]. They live under the ground in branched tunnels they dig using their powerful front teeth. Mole rats are truly blind. Their small eyes are completely covered by a layer of skin [8]. Because of its specific way of life the mole rat was used as experimental animals in studies of biology, immunology, microbiology, physiology and they are interesting also for anatomical studies of morphology and vascularisation of their organs. Available literature offers very little information on the morphology of the organs of this rodent.

The liver lobes, their number and nomenclature, as well as their biliary and vascular system are still the subject of investigation, in several species of the experimental animals, such as the rat $[3,5,19,24]$, rabbit $[12,13,16,18,19]$, guinea pig [19-21], nutria [12], chinchilla $[9,19]$, pig [11], and dog [4, 22]. The only description of the liver in the mole rat was found of Tammam et al. [23], mentioning the nomenclature of the liver lobes, histological structure of the liver as well as the presence of a gallbladder.

Within the rodent species, the mole rat has been less used as an experimental animal in the detailed study of the liver in terms of its lobes, biliary ducts and blood vessels. For this reason, we undertook a detailed basic study on the topography, morphology and vascularisation of the liver in the mole rat.

\section{MATERIALS AND METHODS}

The investigation was carried out on 7 adult mole rats of both sexes ( 3 females and 4 males) which weighed between 180 and $240 \mathrm{~g}$. The mole rats were trapped in Serbia, in the area of south Banat (Deliblato Sands). All animals were found to be clinically healthy.

Being an endangered species, the mole rat is protected by law in the Republic of Serbia. For this reason, the Ministry of Environmental Protection of the Republic of Serbia issued an approval for obtaining the animals from their natural habitats (No. 353-01-1185/2011-03) and the Ethics Committee of the Faculty of Veterinary Medicine in Belgrade issued an approval for conducting this research.

Prior to sacrifice, the animals were sedated with xylazine (Rompur ${ }^{\circledR}$, Bayer, Mississauga/Ontario, Canada) in a dose of $0.1 \mathrm{~mL} / \mathrm{kg}$ intramuscular, and then euthanized with ketamine (Ketamidor 10\%, Richter
Pharma AG, Wels, Austria) in a dose of $0.1 \mathrm{~mL} / \mathrm{kg}$ intramuscular. The topographical position and morphology of livers were studied in the sacrificed animals and preparations preserved in 3\% formaldehyde solution. The biliary system and pancreatic ducts, as well as arterial and venous system of the liver were investigated in injected preparations and roentgenograms. In order to obtain preparations with arterial vascularisation of the liver, after bleeding out stained gelatin was injected into the abdominal aorta. After $24 \mathrm{~h}$ the arterial blood vessels were prepared and photographed with a digital camera. A mixture of red-coloured lead oxide and linseed oil was used for roentgenograms. This mixture was injected into the portal system through the portal vein (PV) before its entry into the liver, and into the liver veins through the caudal vena cava previously tied cranially to the diaphragm. This mixture also was injected into the biliary system through the gallbladder, and into the system of pancreatic ducts through the duodenum, which, in both cases, had been tied off previously. The injected pancreatic ducts, venous blood vessels and biliary system of the liver were X-rayed.

Latin terms were used in accordance with Nomina Anatomica Veterinaria, 2017 [7].

\section{RESULTS}

The liver of the mole rat was located in the intrathoracic part of the abdominal cavity, with the exception of ventral part of the left hepatic lobe which was located on the ventral abdominal wall in the regio xiphoidea, somewhat left to the median plane. On the right of the intrathoracic part of the abdominal cavity the liver was situated between the diaphragm, descending duodenum, jejunum and right kidney, and on the left between the diaphragm, stomach, jejunum and dorsal end of the spleen.

The parietal surface of the liver was strongly convex and it was in contact with diaphragm.

\section{Liver lobes}

Deep fissures divided the liver into five lobes as follow: left, quadrate, right medial, right lateral and caudate lobes.

The left lobe (LS, lobus hepatis sinister) (Fig. 1A, B-1) was the largest and undivided lobe of the liver. It was positioned in the left intrathoracic part of the abdominal cavity with the exception of a small ventral part of this lobe which rests upon the ventral 


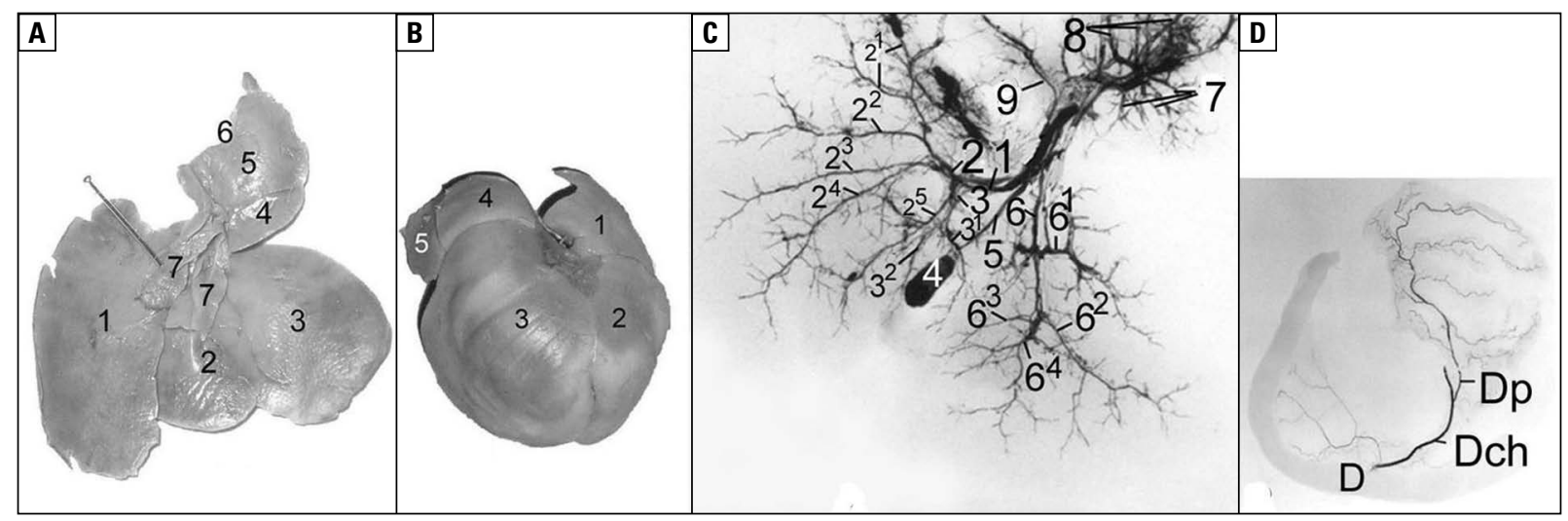

Figure 1. Visceral (A), parietal surface of the liver (B), bile ducts (C) and the common bile duct (D) of the liver in the mole rat; $A$ and B: 1 lobus hepatis sinister; 2 - lobus quadratus; 3 - lobus hepatis dexter medialis; 4 - lobus hepatis dexter lateralis; 5 - processus caudatus; 6 - impressio renalis; 7 - processus papillaris; C: 1 -d. lobi sinistri et lobi quadrati; 2 d. lobi sinistri; $2^{1-5}-$ rr. lobi sinistri; $3-\mathrm{d}$. lobi quadrati; $3^{1-2}-$ rr. lobi quadrati; 4 - vesica fellea; $5-\mathrm{d}$. cysticus; 6 -d. lobi dextri medialis; $6^{1-4}-\mathrm{rr}$. lobi dextri medialis; 7 rr. lobi dextri lateralis; 8 - rr. processi caudati; $9-$ d. processi papillaris; D: D - duodenum; Dp - d. pancreaticus; Dch — d. choledochus.

abdominal wall, in the regio xiphoidea. The stomach overlapped almost the whole visceral surface of the left lobe with the exception of a small ventral portion of that lobe, resting on the jejunum. The left lobe of the liver was covered on the parietal surface by the quadrate lobe.

The quadrate lobe (LQ, lobus quadratus) (Fig. 1A, $B-2)$ was situated on the left in the intrathoracic part of the abdominal cavity. It was separated from the left and right medial liver lobes by deep notches. The quadrate lobe was divided into two components by a deep notch. Its visceral surface rested upon the parietal surface of the left lobe and these lobes partly overlapped each other (Fig. 1B-1, 1B-2). The quadrate lobe was attached to the gallbladder.

The right lobe ( $\mathrm{RL}$, lobus hepatis dexter) was positioned completely in the right intrathoracic part of the abdominal cavity. It was divided into medial (RML) and lateral (RLL) lobes by a deep fissure. The RML (Fig. 1A, B-3) was the second largest lobe of the liver. On the parietal surface from the medial border of the RML, the falciforme ligament appeared as a thin fold that connected the liver to the diaphragm. Parietal surface of the right lateral lobe was partially overlapped by visceral surface of the right medial lobe (Fig. 1B-4, 1B-3).

The caudate lobe ( $\mathrm{CL}$, lobus caudatus) was situated dorsal to the portal fissure and it was divided into a caudate and a papillary process.

The caudate process (CP, processus caudatus) (Fig. $1 \mathrm{~A}, \mathrm{~B}-5)$ was almost overlapped by the visceral surface of the right lateral lobe. Its caudodorsal edge showed very marked imprint for the right kidney (Fig. 1A-6).
The papillary process (PP, processus papillaris) (Fig. 1A-7) was divided by a deep fissure into two nar row and long portions.

The gallbladder (GB, vesica fellea) (Fig. 1C-4) lay in a slight depression on the quadrate lobe. It was cylindrical shaped and it did not reach the ventral border of the liver.

The cystic duct (CD, ductus cysticus) (Fig. 1C-5) was a short duct, 9 to $10 \mathrm{~mm}$ long. It joined the left hepatic duct. The left hepatic duct drained the left and quadrate lobe by one bile duct (Fig. 1C-1). The right hepatic duct was formed by the confluence of the ducts that drained the caudate process of the caudate lobe with the duct that drained the right lateral lobe, than from the duct that drained papillary process of the caudate lobe and the last duct drained the right medial lobe.

The common bile duct (CBD, ductus choledochus) (Fig. 1D-Dch) was formed by the union of the right and left hepatic ducts. It entered the duodenum 6.5 to $7 \mathrm{~mm}$ caudal of the pylorus. A single pancreatic ducts joined lobar and lobular ducts of the pancreas and joined the common bile duct before it entered the duodenum.

\section{The proper hepatic artery (a. hepatica propria)}

The liver of the mole rat was supplied with blood by the proper hepatic artery (PHA), a branch of the hepatic artery (HA, a. hepatica). At the porta hepatis, the PHA (Fig. 2A-I) bifurcated into the right (Fig. 2A-II) and left (Fig. 2A-III) branches. The right branch of PHA first gave off the common branch to the right lateral lobe and caudate process of the caudate lobe 


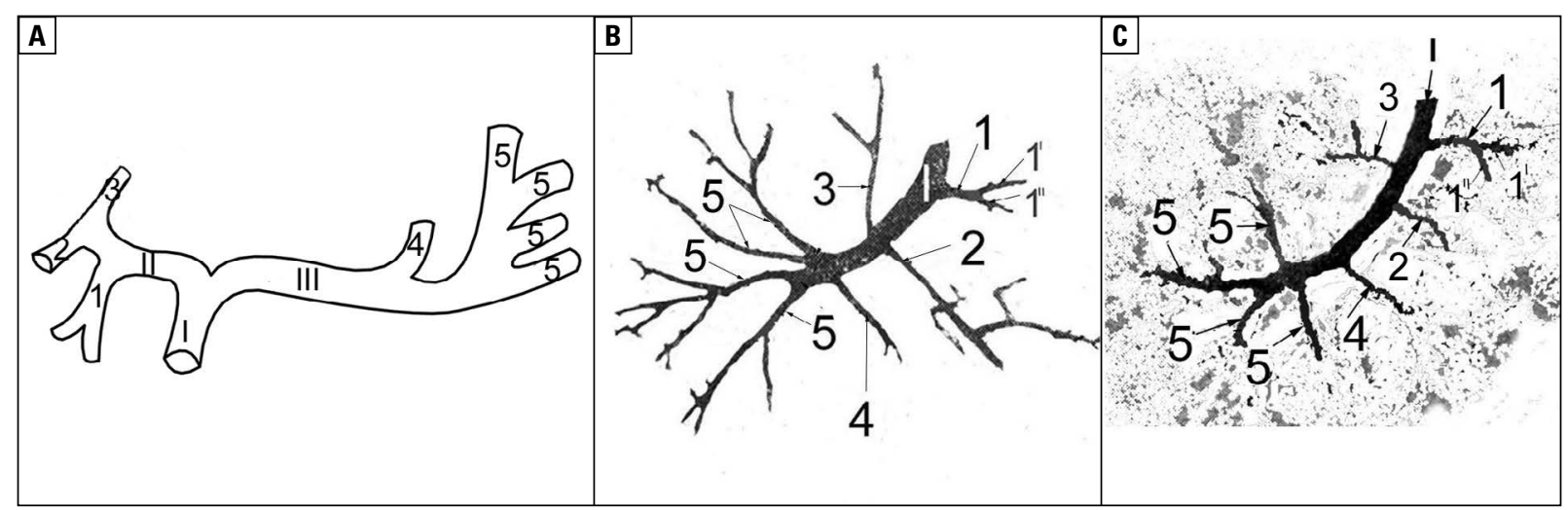

Figure 2. Schematic representation of the intrahepatic branches of the proper hepatic artery $(\mathbf{A})$, radiograph of intrahepatic branches of the portal vein (B) and hepatic veins (C) in the mole rat; A: I - a. hepatica propria; II - r. dexter; III - r. sinister; 1 - r. lobi dextri lateralis et processi caudati; 2 - r. lobi dextri medialis; 3 -r. processi papillaris; 4 - r. lobi quadrati; 5,5,5,5 - rr. lobi sinistri; B: I - v. portae; 1 r. lobi dextri lateralis et processi caudati; 2 r. lobi dextri medialis; 3 -r. processi papillaris; 4 -r. lobi quadrati; $5,5,5,5-$ rr. lobi sinistri; C: I - v. cava caudalis; 1 - v. hepatica lobi dextri lateralis et processi caudati; 2 - v. hepatica lobi dextri medialis; 3 - v. hepatica processi papillaris; $4-v$. hepatica lobi quadrati; $5-v$. hepatica lobi sinistri.

(Fig. 2A-1), then the branch to the right medial lobe (Fig. 2A-2) and last branch to the papillary process of the caudate lobe (Fig. 2A-3). The left branch of PHA first gave off the branch that supplied the quadrate lobe (Fig. 2A-4) and then it branched into four larger arterial branches that supplied the left lobe (Fig. 2A-5).

\section{The portal vein (v. portae)}

The first portal branch of the common portal vein (CPV) (Fig. 2B-I) was very short and it immediately was divided into two branches, one to the caudate process of the caudate lobe and one to the right lateral lobe (Fig. 2B-1). From its ventral wall, CPV gave off two separated branches to the right medial (Fig. 2B-2) and quadrate lobe (Fig. 2B-4). From the dorsal wall, CPV gave off a portal branch to the papillary process of the caudate lobe that ramified into both portion of the papillary process (Fig. 2B-3). Between the left and quadrate lobes the CPV divided into four final branches (Fig. 2B-5) supplying the dorsal, middle and ventral portion of the left lobe.

\section{The hepatic veins ( $v v$. hepaticae)}

Eight large hepatic veins (HVs) drained the blood from the lobes of the liver into the caudal vena cava (Fig. 2C-I) during its passage through the liver. The caudate processes of the caudate lobe and right lateral lobe were drained by two separated HVs united by a common HV (Fig. 2C-1) that opened into the caudal vena cava. The right medial lobe (Fig. 2C-2), papillary process of the caudate lobe (Fig. $2 \mathrm{C}-3$ ) and quadrate lobe (Fig. 2C-4), were drained by three separated HVs that opened into the caudal vena cava separately. The left lobe was drained into the caudal vena cava by four HVs (Fig. 2C-5).

\section{DISCUSSION}

Knowledge of the nomenclature of liver lobes was of great importance for understanding the vascular and biliary branching pattern in the liver of experimental animals.

There has been no study in the literature describing the liver and intrahepatic vascular and biliary system of the mole rat. Only topographical position and names of the liver lobes were mentioned by Tammam et al. [23] in mole rats. For this reason, our study is the first study on the topography, morphology, intrahepatic ramification of the artery, veins and bile ducts of mole rats (Spalax leucodon) described and illustrated in detail.

A comparable topography of the liver of rats, rabbits, guinea pigs and chinchillas was described by Stan [19]. The livers of those animals were positioned almost in intrathoracic part of the abdominal cavity. Tammam et al. [23], in the study on the histological structure of the digestive tract of mole rats, including the liver, mentioned that the liver of this rodent was located in the intrathoracic part of the abdominal cavity. However, our study suggested the presence a small ventral portion of the left lobe located on the ventral abdominal wall in the regio xiphoidea, which was not described in mole rats by Tammam et al. [23]. This portion of left lobe is an accessible 
place for puncture of the liver and further laboratory analysis of this organ.

The liver of mole rats was lobulated as found in experimental small mammals $[5,9,12,18,21]$ and some domestic mammals, such as pigs and dogs [11, 22]. Stan [19] compared the division of the liver into lobes among rabbits, guinea pigs and chinchillas, and showed that six liver lobes in guinea pigs (left lateral and medial lobes, quadrate lobe, right medial and lateral lobes and caudate lobe) corresponded to that in nutria liver [12]. The same number of lobes was described by Osman et al. [11] in pigs and by Swielim [22] in dogs. In rabbits the right lobe was single $[13,18,19]$. The quadrate lobe recognised by Stamatova-Yovcheva et al. [18] in rabbits, Stan et al. [21] in guinea pigs and Osman et al. [11] in pigs was very small and was not observed on the diaphragmatic surface of the liver in these animals.

The basic nomenclature of the rat liver lobes was not uniform. Kogure et al. [3], Martins and Neuhaus [5] and Quesenberry [14] described four lobes of the liver in rats (left lateral, middle, caudate and right lobes) and Vdoviaková et al. [24] merged the left lateral lobe with the middle lobe into one lobe, thus the rat liver was divided into the left lateral and medial lobes, right medial and lateral lobes and caudate lobe divided into two portions. The middle lobe of rats, described by Martins and Neuhaus [5] divided into the left medial and right medial lobes, was not detected in our study.

Tammam et al. [23] in the study on morphology of the liver in mole rats did not explain according to which nomenclature the liver was divided into: left, right, right central, spigelian and caudate lobes. In our study, the division of the liver into left, quadrate, right medial, right lateral and caudate lobes was adopted according to Nomina Anatomica Veterinaria 2017 [7].

The presence of the GB in small mammals was variable and its morphology was mainly dependent upon diet [10]. The most species of the experimental animals, except rats, had a well-developed GB, located in the shallow depression on the quadrate or the right medial lobes [18-20]. The presence of a gallbladder on the right medial lobe of the liver described by Tammam et al. [23] in mole rats was not confirmed in our study in which the GB was attached to the quadrate lobe.

Among laboratory animals, rats are the most frequently used as animal models in biomedical research.
Detailed knowledge of the intrahepatic vascular and biliary anatomy and also their variations are of the great importance for experimental hepatobiliary surgery and transplantation of the liver [15]. Previous anatomical studies described vascular and biliary anatomy of the liver in rats $[3,5,15,24]$ and the obtained data were compared with those of humans.

In the literature there have been no studies describing the vascular and biliary branching pattern in the liver of mole rats.

The liver of different species of experimental animals was supplied by the PHA or HA and their division into the right and left branches was described by Martins and Neuhaus [5] and Vdoviaková et al. [24] in rats, Stamatova-Yovcheva et al. [17] in rabbits and Osman et al. [11] in pigs. Stamatova-Yovcheva et al. [17] asserted that in rabbits PHA first supplied the caudate lobe and its continuation was HA that bifurcated into the right and left branches. In rats $[5,24]$ and rabbits [17] the caudate and right (lateral and medial) lobes were supplied by branches from the right branch of HA. The same lobes supplied by r. dexter lateralis and medialis were described by Osman et al. [11] in pigs and by Swielim [22] in dogs. The left lateral, left medial and quadrate lobes were supplied by branches from the left branch of PHA in rats [24] and branches of HA in rabbits [17].

The presence of the PV in the porta $(\mathrm{PH}$, porta hepatis) and its division into two main branches, right and left, was described by Sänger et al. [15], Vdoviaková et al. [24] in rats, Stamatova-Yovcheva et al. [17] in rabbits and Mari and Acocella [4] in dogs. However, Osman et al. [11] mentioned that in pigs, PV was divided into R. dexter dorsalis, $R$. dexter ventralis and left branch. The division of the PV into the main branches was not detected in our study.

Kogure et al. [3] and Martins and Neuhaus [5] recorded that in rats each liver lobe had their own lobar portal branch. In studies on rabbits, Stamatova-Yovcheva et al. [17] showed that the right portal branch supplied only right lobe. Similar results were obtained by Vdoviaková et al. [24] in rats where right portal branch supplied the caudate, right lateral and right medial lobes. The same lobes in pigs were supplied by $r$. dexter lateralis, as described by Osman et al. [11]. All of the above authors agreed that the left lobes and quadrate lobe of the liver in rats, rabbits, and pigs were supplied by branches from the left branch of PV. 
Numerous authors have studied hepatic venous system of the liver of mammals $[4,5,11,16,17]$. It has been reported that both portion of the caudate lobe in rats $[5,24]$ was drained into the caudal vena cava (CVC) by one HV. Similar results were reported in rabbits by Stamatova-Yovcheva et al. [17]. However, in dogs, the caudate lobe was drained by two HVs, as described by Mari and Acocella [4], of which the vein from the caudate process entered directly and the vein from the papillary process indirectly through the main lobar vein, into the CVC.

In rats, both portions of the right lobe were drained into the CVC by one HV $[5,15]$. Such results were found by Mari and Acocella [4] in dogs. The undivided right lobe of rabbits described by Seo et al. [16] was drained into CVC by two separated veins. In the present study both processes of the caudate lobe and both lobes of the right lobe had a different vascular blood supply.

The quadrate lobe was drained indirectly through the middle $\mathrm{HV}$ into the $\mathrm{CVC}$, as described in rabbits by Seo et al. [16], and through the main HV, as reported in dogs by Mari and Acocella [4]. In the present study the quadrate lobe was drained into CVC by one HV.

The left lateral lobe in rats $[3,5,15]$, rabbits [17] and dogs [4] was drained into CVC by one HV. Also, in rabbits and dogs the left medial lobe had one HV.

In the present study four HVs drained blood from the left lobe.

The extrahepatic biliary tract of the experimental animals has been the subject of research by numerous authors, and studies on their intrahepatic bile branching system have been scarce. At the literature, there was no study on the biliary system of mole rats. The presence of the CBD, common hepatic duct (CHD) and $C D$ in experimental animals were described by many authors $[5,17,20]$. The formation of the CBD by the union of the right and left hepatic ducts revealed in our study was also reported in guinea pigs by Stan [20]. However, the union of the right and left hepatic ducts resulting in a CHD has been described in rabbits by Seo et al. [16] and Stamatova-Yovcheva et al. [17]. The results observed in chinchillas by Nowak et al. [9] have shown that two to three cystic ducts formed a network joined with the hepatic duct system into complex system of bile ducts.

The presence of CBD formed by the union of the CD and CHD was reported in pigs by Osman et al. [11]. Rats have neither a GB nor a CD. In rats, all lobar bile ducts fused into the CHD, so that on its course to the duodenum it was completely imbedded in the pancreas [5]. The CBD and the pancreatic duct opened into the duodenum separately in guinea pigs, rats and chinchillas $[1,5,9]$.

The presence of the right and left hepatic ducts in experimental mammals was described by numerous authors $[11,17,24]$.

The presence of the right hepatic duct to drain right and caudate lobes was described by Stamatova-Yovcheva et al. [17] in rabbits and by Vdoviacová et al. [24] in rats. Similar results were obtained by Stan [20] in guinea pigs. Osman et al. [11] in pigs and Coq [2] in dogs mentioned that the right hepatic duct was formed by the confluence of ducts from the caudate process and the right lateral and right medial lobes. The left hepatic duct draining the left (lateral and medial) and quadrate lobes were noted by Stan [20] in guinea pigs, Vdoviacová et al. [24] in rats and Stamatova-Yovcheva et al. [17] in rabbits.

\section{CONCLUSIONS}

The macroscopic anatomy of the liver and its vascular and biliary system of mole rats was similar to that of other experimental animals, and identified differences may be related to the adaptation to the mode of life and nature of diet in this rodent.

\section{Conflict of interest: None declared}

\section{REFERENCES}

1. Cai WQ, Gabella G. Innervation of the gall bladder and biliary pathways in the guinea-pig. J Anat. 1983; 136(Pt 1): 97-109, indexed in Pubmed: 6833124.

2. Coq P. La segmentation hepatique des carnivores. Rec Med Vet. 1965; 141: 234-268.

3. Kogure $K$, Ishizaki $M$, Nemoto $M$, et al. A comparative study of the anatomy of rat and human livers. J Hepatobiliary Pancreat Surg. 1999; 6(2): 171-175, doi: 10.1007/ s005340050101, indexed in Pubmed: 10398905.

4. Mari L, Acocella F. Vascular anatomy of canine hepatic venous system: A basic for liver surgery. Anat Histol Embryol. 2015; 44: 212-224.

5. Martins PN, Neuhaus P. Surgical anatomy of the liver, hepatic vasculature and bile ducts in the rat. Liver Int. 2007; 27(3): 384-392, doi: 10.1111/j.1478-3231.2006.01414.x, indexed in Pubmed: 17355461.

6. Méhley L. Species generis Spalax, a földi kutyák fajai. Szarmazès ès rendszertani takinètben. A Madyar Tudomanyos Akademia, Kiadasa, Budapest 1969.

7. Nomina Anatomica Veterinaria, sixth edition. Prepared by the International Committee on Veterinary Gross Anatomical Nomenclature (I.C.V.G.A.N.). Editorial Committee Hannover, Ghent, Columbia, MO (U.S.A.), Rio de Janeiro 2017. 
8. Nowak RM. Walker's Mammals of the World. The Johns Hopkins University Press, Baltimore, USA; London, United Kingdom 1999.

9. Nowak E, Kuchinka J, Szczurkowski A, et al. Extrahepatic biliary tract in chinchilla (Chinchilla laniger, Molina). Anat Histol Embryol. 2015; 44(3): 236-240, doi: 10.1111/ ahe.12137, indexed in Pubmed: 25091180.

10. Oldham-Ott C, Gilloteaux J. Comparative morphology of the gallbladder and biliary tract in vertebrates: Variation in structure, homology in function and gallstones. Microsc Res Tech. 1997; 38(6): 571-597, doi: 10.1002/(sici)10970029(19970915)38:6<571::aid-jemt3>3.0.co;2-i.

11. Osman F, Wally Y, El-Nady F, et al. Gross anatomical studies on the portal vein, hepatic artery and bile duct in the liver of the pig. J Vet Anat. 2008; 1(1): 59-72, doi: 10.21608/ jva.2008.45456.

12. Pérez W, Lima M. Anatomical Description of the Liver, Hepatic Ligaments and Omenta in the Coypu (Myocastor coypus). Int J Morphol. 2007; 25(1), doi: 10.4067/s071795022007000100007.

13. Pérez W, Möller R, Martin E. Peritoneal folds of the rabbit (Oryctolagus cuniculus). Anat Histol Embryol. 2005; 34(3): 167-170, doi: 10.1111/j.1439-0264.2005.00587.x, indexed in Pubmed: 15929731.

14. Quesenberry K. Ferrets, rabbits and rodents: Clinical Medicine and Surgery. 3th ed. Elsevier, St. Louis, Missouri, USA 2012.

15. Sänger $C$, Schenk $A$, Schwen LO, et al. Intrahepatic vascular anatomy in rats and mice - variations and surgical implications. PLoS One. 2015; 10(11): e0141798, doi: 10.1371/ journal.pone.0141798, indexed in Pubmed: 26618494.

16. Seo TS, Oh JH, Lee DH, et al. Radiologic anatomy of the rabbit liver on hepatic venography, arteriography, portog- raphy, and cholangiography. Invest Radiol. 2001; 36(3): 186-192, doi: 10.1097/00004424-200103000-00008, indexed in Pubmed: 11228583.

17. Stamatova-Yovcheva K, Dimitrov R, Dilek GO. Radiographic study of the topography of the hepatic vasculature and bile ducts of the rabbit. Bulg J Agric Sci. 2018; 24(3): 497-502.

18. Stamatova-Yovcheva K, Dimitrov R, Kostov D, et al. Anatomical macromorphological features of the liver in domestic rabbit (Oryctolagus cuniculus). J Sci. 2012; 10: 85-90.

19. Stan F. Comparative study of the liver anatomy in the rat, rabbit, guinea pig and chinchilla. Bulletin UASVM Veterinary Medicine. 2018; 75(1): 33, doi: 10.15835/ buasvmcn-vm:002717.

20. Stan F. Macroscopic anatomy of the gallbladder and extrahepatic biliary tract in the guinea pig (Cavia porcellus). Scientific Works. Series C. Veterinary Medicine. 2017; 43(2): 25-29.

21. Stan F, Martonos C, Dezdrobitu C, et al. Detailed morphological description of the liver and hepatic ligaments in the guinea pig (Cavia porcelus). Scientific Works. Series $C$. Veterinary Medicine. 2017; 43(1): 35-41.

22. Swielim GEA. Intrahepatic distribution of the portal vein and hepatic vessels in the dog. A lex. J Vet Sci. 1989; 5(2): 33-45, doi: 10.21608/jva.2008.45456.

23. Tammam O, Emara S, Sedera DA. Some histological studies on the digestive system of the spalax leucodon (lesser blind mole rat) inhabit the egyptian north coast. J Environ Studies Res. 2017; 6((E2)): 124-135, doi: 10.21608/ jesr.2017.57278.

24. Vdoviaková K, Vdoviaková K, Petrovová E, et al. Importance Rat Liver Morphology and Vasculature in Surgical Research. Med Sci Monit. 2016; 22: 4716-4728, doi: 10.12659/msm.899129, indexed in Pubmed: 27911356. 